\title{
Modeling of a high frequency ultrasonic transducer using periodic structures
}

\author{
P. Maréchal ${ }^{1}$, L. Haumesser ${ }^{1}$, G. Feuillard ${ }^{1}$, L.P. Tran-Huu-Hue ${ }^{1}$, J. Holc $^{2}$, D. Kuščer ${ }^{2}$, M. Lethiecq ${ }^{1}$ \\ ${ }^{1}$ François-Rabelais University, LUSSI, FRE 2448 CNRS, ENIVL, rue de la Chocolaterie, Blois, France \\ ${ }^{2}$ Joseph Stefan Institute, Jamova 39, Ljubljana, Slovenija
}

\begin{abstract}
Thick film technology has been used to produce high frequency $(>20 \mathrm{MHz})$ transducers. Alumina or silicon substrates appear to be inappropriate as backing material because of their low attenuation and high acoustic impedance. Alternatively, porous materials have a sufficient attenuation but their acoustic impedance lowers the sensitivity. In this context, the use of a Bragg cell between the piezoelectric film and the substrate is investigated for high frequency ultrasonic transducers for non destructive testing or medical applications. A recursive transmission/reflection model is presented. The influence of the constitutive layer properties of a Bragg cell is studied for two compatible sets of materials $\left(\mathrm{Au} / \mathrm{PZT}\right.$ or $\left.\mathrm{Pt} / \mathrm{Al}_{2} \mathrm{O}_{3}\right)$. This model is then used to highlight the interest of a periodic structure loaded with a substrate. Finally, experimental results on a Bragg cell are reported and discussed.
\end{abstract}

Key words: periodic structures, piezoelectric transducer, high frequency.

\section{A. Introduction}

The elaboration of thick-film high frequency ultrasonic transducers requires a compromise between theoretical design and fabrication possibilities in terms of material properties and sizes. All the substrates adapted to the fabrication process have an acoustic impedance close to that of the active film or even higher, leading to a strong damping of the piezoelectric resonance. Therefore, it is necessary to adapt the input impedance at the rear face of the piezoelectric layer to avoid excessive lowering of its resonance frequency and of transducer sensitivity. Here, the proposed solution is the insertion of a Bragg cell between the piezoelectric layer and the substrate. Available material combinations are restricted by thermal and chemical compatibility. Thus, the ratio of acoustic impedance between the two layers constituting the Bragg cell is limited. First, the recursive model and related formulas for the reflection and transmission coefficients are presented without restriction to periodicity. Then, the case of a Bragg cell on a substrate is presented as a way to modify the input impedance of the backing. Second, the influence of the acoustic impedance ratio on the transmission coefficient is presented and two available compatible sets of materials $\left(\mathrm{Au} / \mathrm{PZT}\right.$ or $\left.\mathrm{Pt} / \mathrm{Al}_{2} \mathrm{O}_{3}\right)$ are discussed. The influence of the number of layers is discussed both in terms of minimum value and bandwidth. Then, the high frequency transducer design is presented, using a recursive calculation of the input impedance of the backing. Finally, experimental results are presented for a Bragg cell made of $\mathrm{Au} / \mathrm{PZT}$.

\section{B. Modeling}

The modeling of a periodic structure is developed here on the basis of a one dimensional assumption. It takes into account multiple reflections between the interfaces.

\section{B.1. Reflected and transmitted pressure fields}

Using the notation proposed by Conoir [1], the Fresnel reflection and transmission coefficients, $R$ and $T$, at the interface between layers indexed $n$ and $n+1$ are:

$$
\begin{aligned}
& R_{n, n+1}=\frac{Z_{n}-Z_{n+1}}{Z_{n}+Z_{n+1}} e^{+2 j \varphi_{n}}, \\
& T_{n, n+1}=\frac{2 Z_{n+1}}{Z_{n+1}+Z_{n}} e^{-j\left(\varphi_{n+1}-\varphi_{n}\right)},
\end{aligned}
$$

reciprocally,

$$
\begin{aligned}
R_{n+1, n} & =\frac{Z_{n+1}-Z_{n}}{Z_{n+1}+Z_{n}} e^{-2 j \varphi_{n+1}}, \\
\text { and } \quad T_{n+1, n} & =\frac{2 Z_{n}}{Z_{n}+Z_{n+1}} e^{-j\left(\varphi_{n+1}-\varphi_{n}\right)}
\end{aligned}
$$

where $Z_{n}$ is the acoustic impedance, $\varphi_{n}=k_{n} z_{n}=\omega z_{n} / c_{n}$ and $\varphi_{n+1}=k_{n+1} z_{n}=\omega z_{n} / c_{n+1}$ are the phases expressed as a function of the interface position $z_{n}$, wave number $k_{n}$, (angular pulsation $\omega$ and longitudinal wave velocity $c_{n}$ ).

The reflection coefficient of a multilayer structure is calculated from layer $n=N$ to layer 1 :

$$
\begin{array}{ll} 
& R=R_{12}+\frac{T_{12} \rho_{23} T_{21}}{1-R_{21} \rho_{23}}, \\
\text { where } & \rho_{n, n+1}=R_{n, n+1}+\frac{T_{n, n+1} \rho_{n+1, n+2} T_{n+1, n}}{1-R_{n+1, n} \rho_{n+1, n+2}}, \\
\text { for } \quad & 2 \leqslant n \leqslant N \text { and } \rho_{N+1, N+2}=R_{N+1, N+2} .
\end{array}
$$

The transmission coefficient of a multilayer structure is calculated similarly from layer $n=1$ to layer $N$ :

$$
\begin{array}{ll} 
& T=T_{12} \prod_{n=2}^{n=N} \frac{T_{n, n+1}}{1-R_{n, n+1} \rho_{n, n-1}}, \\
\text { where } & \rho_{n+1, n}=R_{n+1, n}+\frac{T_{n+1, n} \rho_{n, n-1} T_{n, n+1}}{1-R_{n, n+1} \rho_{n, n-1}}, \\
\text { for } \quad & 2 \leqslant n \leqslant N \text { and } \rho_{21}=R_{21} .
\end{array}
$$


This formalism is then used to model periodic structures for high frequency transducer applications (Fig.1).

Bragg cell

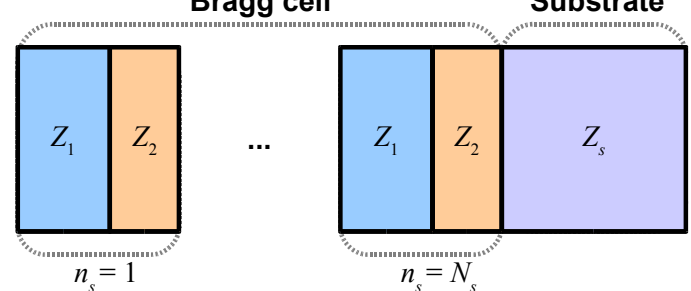

Fig.1: Periodic structure made of $N_{s}$ elementary stacks of $N_{\text {layer } s}$ $=2$ layers loaded with a substrate.

Throughout the study, the layers constituting the periodic structure will be considered quarter wavelength thick.

\section{B.2. Influence of the constitutive layers}

\section{B.2.1. Ratio of acoustic impedance}

The transmission coefficient of an elementary stack is calculated for various impedance ratios as a function of normalized frequency (Fig.2 (a)). The higher the impedance mismatch, the lower is the transmission coefficient. Fig.2 (b) shows the evolution from 0 (total reflection) up to 1 (total transmission) as a function of the impedance ratio. Vertical lines indicate two sets of materials compatible with thick film technology, the properties of which are listed in Table 1. For a chosen ratio of acoustic impedance $Z_{2} / Z_{1}<1$, an elementary $\mathrm{Au} / \mathrm{PZT}$ stack with $Z_{2} / Z_{1}=0.54$ (Fig.3 (a)) or Pt/ $\mathrm{Al}_{2} \mathrm{O}_{3}$ stack with $Z_{2} / Z_{1}=0.25$ (Fig.3 (b)) leads to decrease the transmission coefficient around the center normalized frequency $f / f_{0}=1$.

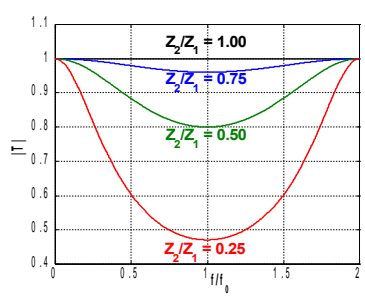

a)

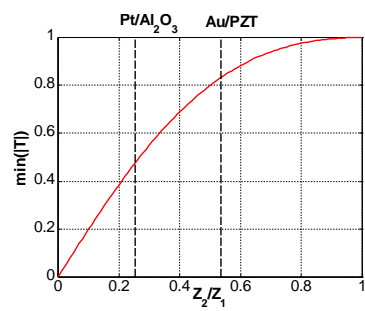

b)
Fig.2: (a) Transmission coefficient as a function of the normalized frequency $f l f_{0}$ for $Z_{2} / Z_{1}=0.25,0.5,0.75$ and 1 and (b) minimum transmission coefficient as a function of $Z_{2} / Z_{1}$ for a structure constituted of a single stack.

Table 1: Acoustic properties of the basic stack materials

\begin{tabular}{cccc}
\hline Set of materials & $Z_{1}(\mathrm{MRa})$ & $Z_{2}(\mathrm{MRa})$ & $Z_{2} / Z_{1}$ \\
\hline $\mathrm{Au} / \mathrm{PZT}$ (dense) & 63.8 & 34.2 & 0.54 \\
\hline $\mathrm{Pt} / \mathrm{Al}_{2} \mathrm{O}_{3}$ (porous) & 69.8 & 17.7 & 0.25 \\
\hline $\begin{array}{c}Z_{1} \text { and } Z_{2}: \text { Acoustic impedance of the layers constituting the } \\
\text { basic stack; } Z_{2} / Z_{1}: \text { Ratio of acoustic impedance. }\end{array}$
\end{tabular}

\section{B.2.2. Number of stacks}

For the selected sets of materials, an increase of the number of elementary stacks $N_{s}$ (Fig.3) has two effects on the transmission coefficient as a function of frequency. First, the number of local maxima of the transmission coefficient $\max (|T|)=1$ from $f / f_{0}=0$ to 1 equals the number of stacks $N_{s}$. Second, as the number of stacks $N_{s}$ increases, the minimum value of the transmission coefficient $\min (|T|)$ tends to zero and the relative bandwidth converges.

a)

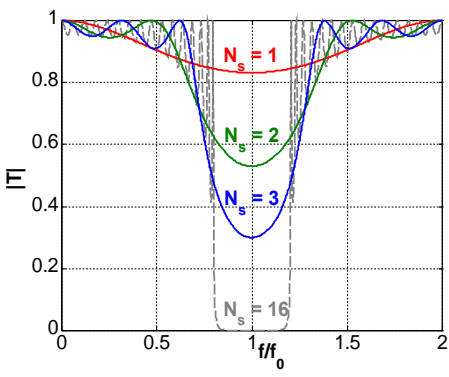

b)

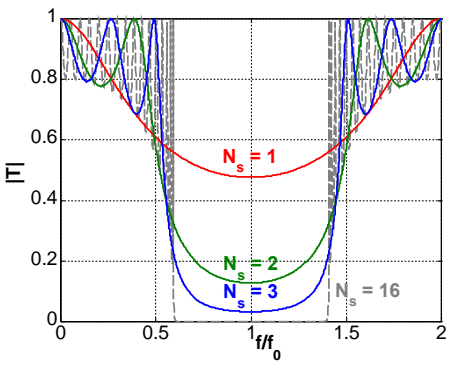

Fig.3: Transmission coefficient as a function of normalized frequency $f / f_{0}$ for a basic stack (a) Au/PZT $\left(Z_{2} / Z_{1}=0.54\right)$ and (b) $\mathrm{Pt} / \mathrm{Al}_{2} \mathrm{O}_{3}\left(Z_{2} / Z_{1}=0.25\right)$ with number of stacks $N_{s}=1,2,3$ and 16.

\section{B.2.3. Stop-band characteristics}

The minimum value of the transmission coefficient $\min (|T|)$ (Fig.4 (a)) and the relative bandwidth $B W_{r}$ (Fig.4 (b)) are represented as a function of the number of stacks, from $N_{s}=1$ to 16 .

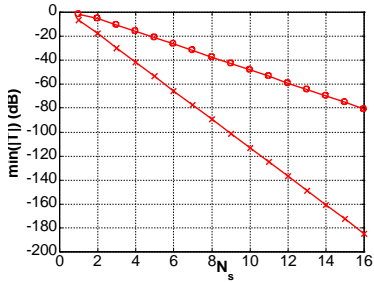

a)

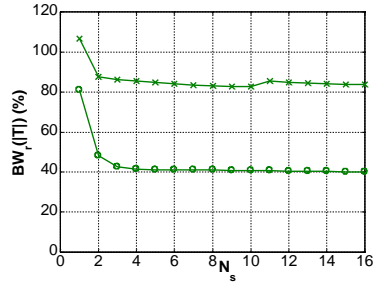

b)
Fig.4: (a) Minimum transmission coefficient and (b) relative bandwidth as a function of the number of stacks $N_{s}=1$ to 16 , for $\mathrm{Au} / \mathrm{PZT}$ (circle) and $\mathrm{Pt} / \mathrm{Al}_{2} \mathrm{O}_{3}$, (cross) respectively.

These parameters strongly depend on the impedance ratio and number of stacks. As the number of stacks $N_{s}$ increases, the transmission coefficient $\min (|T|)$ tends towards 0 and the relative bandwidth $B W_{r}$ towards 0.4 and 0.82 for $\mathrm{Au} / \mathrm{PZT}$ and $\mathrm{Pt} / \mathrm{Al}_{2} \mathrm{O}_{3}$, respectively. The bandwidth of the stop-band directly depends on the ratio of acoustic impedances $Z_{2} / Z_{1}$ [2]. Reducing the impedance ratio leads to a decrease of the transmission coefficient and of the bandwidth of the stop-band. Therefore, the minimum transmission coefficient $\min (|T|)$ and relative bandwidth $B W_{r}$ are fitted in the practical range $0.2<Z_{2} / Z_{1}<0.8$, here for $N_{s}=4$ stacks:

and

$$
\min (|T|)=10^{-4\left(Z_{2} / Z_{1}\right)^{2}+8\left(Z_{2} / Z_{1}\right)-4}
$$$$
B W_{r}=2\left(1-\left(Z_{2} / Z_{1}\right)^{0.4}\right)
$$ 


\section{B.3. Input acoustic impedance}

On the basis of previous results [3], the complex input acoustic impedance of the first layer $\left(Z_{\text {in } 1}=\right.$ $Z_{\text {in back }}$ ) of the Bragg cell was calculated recursively from the interface with the substrate $\left(Z_{\text {in }, N+1}=Z_{S}\right)$. The input impedance through one layer is:

$$
Z_{\text {in }, n}=Z_{n} \frac{Z_{\text {in }, n+1}+Z_{n} \operatorname{th}\left(j \theta_{n}\right)}{Z_{n}+Z_{\text {in }, n+1} \operatorname{th}\left(j \theta_{n}\right)}
$$

where $\theta_{n}$ is the phase delay of layer $n$, and $Z_{\mathrm{in}, N+1}=Z_{s}$ is the acoustic load.

Depending on the ratio of acoustic impedance of the layers constituting the basic stack, the input acoustic impedance is either increased $\left(Z_{2} / Z_{1}<1\right)$ or decreased $\left(Z_{2} / Z_{1}>1\right)$. For a quarter wavelength layer, the phase delay at $f f f_{0}=1$ is $\theta_{n}=\pi / 2$ and equation (7) becomes:

$$
\begin{aligned}
Z_{\mathrm{in}, n} & =\frac{Z_{n}^{2}}{Z_{\mathrm{in}, n+1}} \quad \text { for } \quad 1 \leq n \leq N-1 \\
\text { and } \quad Z_{\mathrm{in}, N} & =\frac{Z_{N}^{2}}{Z_{s}}
\end{aligned}
$$

Thus, the global input impedance for $N$ quarter wavelength layers is obtained recursively:

$$
Z_{\text {in }, \text { back }}=Z_{\text {in }, 1}=Z_{s}^{(-1)^{v}} \prod_{n=1}^{n=N} Z_{n}^{2(-1)^{(n+1)}}
$$

Particularly, in the case of a basic stack made of $N_{\text {layers }}=2$ layers, the total number of constitutive layers $N \stackrel{\text { layers }}{=} N_{S} \cdot N_{\text {layers }}$ is even and the input impedance results in:

$$
Z_{\text {in }, \text { back }}=Z_{s} \prod_{n_{s}=1}^{n_{s}=N_{s}}\left(\frac{Z_{1}}{Z_{2}}\right)^{2}=Z_{s}\left(\frac{Z_{1}}{Z_{2}}\right)^{2 N_{s}}
$$

Equation (9) shows the extreme value of the backing input impedance evolving linearly with the acoustic impedance of the substrate, but in power law with the number of stacks for the acoustic impedance ratio.

\section{B.3.1. Stack with $Z_{2} / Z_{1}<1$}

In the case where $Z_{2} / Z_{1}<1$, an elementary stack loaded by a substrate of acoustic impedance $Z_{s}=Z_{2}$ has an input impedance modulus ranging from $\mid Z_{\text {in } \text {,back }}{ }^{s}=Z_{2}$ to $\left|Z_{\text {in, back }}\right|=Z_{1}^{2} / Z_{2}$. Using the properties listed in Table 1 , this maximum value of input impedance rapidly tends to infinity. Its value equals $\left|Z_{\text {in.back }}\right|=119 \mathrm{MRa}$ for a $\mathrm{Au} / \mathrm{PZT}$ elementary stack (Fig.5) and at $\left|Z_{\text {in,back }}\right|=275$ $\mathrm{MRa}$ for a $\mathrm{Pt} / \mathrm{Al}_{2} \mathrm{O}_{3}$ elementary stack.

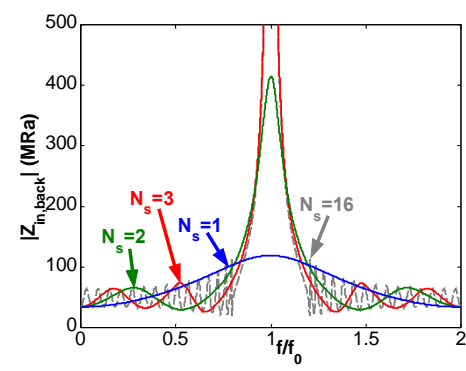

Fig.5: Input impedance modulus at the rear face of the piezoelectric layer for Au/PZT stack on PZT substrate.

\section{B.3.2. Stack with $Z_{2} / Z_{1}>1$}

In the case $Z_{2} / Z_{1}>1$, this stack loaded by a substrate of acoustic impedance $Z_{s}=Z_{1}$ has an input impedance modulus ranging from $\left|Z_{\text {in, back }}^{s}\right|=Z_{1}^{3} / Z_{2}^{2}$ to $\left|Z_{\text {in,back }}\right|=$ $Z_{1}$. The value of the input impedance at the center of the stop-band tends to zero. Using the properties listed in Table 1, this maximum value is $\left|Z_{\text {in, back }}\right|=10 \mathrm{MRa}$ for a PZT/Au elementary stack (Fig.6) and $\left|Z_{i n, b a c k}\right|=1 \mathrm{MRa}$ for a $\mathrm{Al}_{2} \mathrm{O}_{3} / \mathrm{Pt}$ elementary stack.

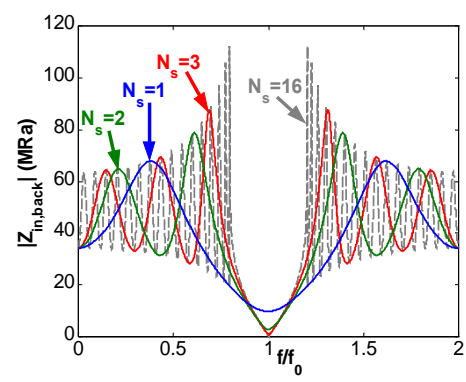

Fig.6: Input impedance modulus at the rear face of the piezoelectric layer for PZT/Au stack on PZT substrate.

This typical configuration allows to lower the input acoustic impedance of the backing. It will be used in section $\mathrm{C}$ to make a reflective substrate in a given frequency range.

\section{B.3.3. Relative bandwidth}

The relative bandwidth of the stop-band $B W_{r}$ in which the modulus of the input impedance of the backing is lower than a chosen threshold can be determined by calculation. It is illustrated for a number of stacks $N_{s}=16$, for both cases where $Z_{2} / Z_{1}<1$ (Fig.7 (a)) and $Z_{2} / Z_{1}>1$ (Fig.7 (b)). These abacuses allow to predict the shape of the modulus of the input impedance for a given set of materials characterized by a given ratio $Z_{2} / Z_{1}$.

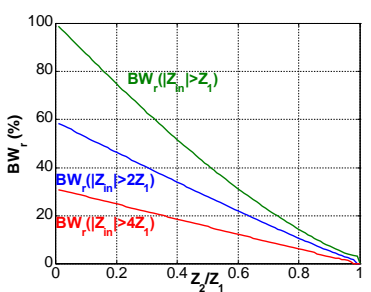

a)

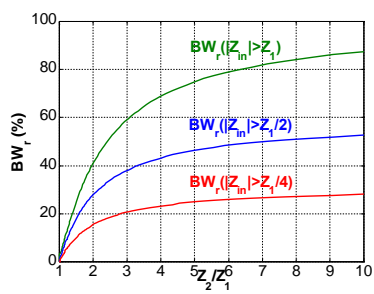

b)
Fig.7: Relative bandwidth of the input impedance at the rear face of the piezoelectric layer for (a) $Z_{2} / Z_{1}<1$ and for (b) $Z_{2} / Z_{1}$ $>1$ acoustic impedance stack, with $N_{s}=16$.

\section{Experimental results}

\section{C.1. Integrated Bragg transducer}

To fabricate a high frequency transducer using this principle, a four stacks integrated Bragg structure was screen-printed. A porous PZT substrate was chosen and several layers of gold and PZT were screen-printed on it to constitute the Bragg cell (Fig.8). Both thickness and acoustic properties (due to variations of porosity) vary significantly. Namely, the thicknesses of the constitutive layers were evaluated around $24 \pm 5$ and $34 \pm 5 \mu \mathrm{m}$ for porous PZT and $\mathrm{Au}$, respectively. 


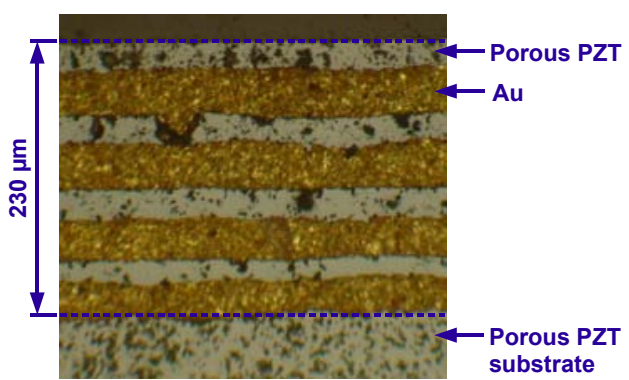

Fig.8: Integrated Bragg structure screen-printed on a porous PZT substrate, constituted of four elementary stacks of Au and porous PZT.

Then, a piezoelectric layer (Pz21) resonating at $f_{a}=36$ $\mathrm{MHz}$ was glued at the top of the Bragg cell to make a transducer. The theoretical input impedance modulus of the integrated Bragg structure on the rear face of the piezoelectric layer is show in Fig.9 (a). The thickness of the glue layer was estimated around $3 \mu \mathrm{m}$, gold and porous PZT properties were taken from literature [4]. The back face electrode and glue layers have modified this input impedance, resulting in $\left|Z_{\text {in,back }}\right|<Z_{s}$ and $|R|=\mid\left(Z_{\text {in }}-\right.$ $\left.Z_{s}\right) /\left(Z_{\text {in }}+Z_{s}\right) \mid>0$ (Fig. $\left.9(\mathrm{~b})\right)$ in a large bandwidth.

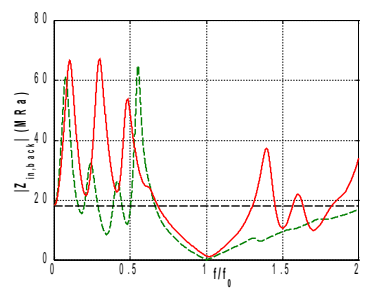

a)

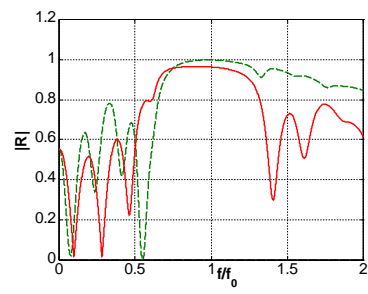

b)
Fig.9: (a) Input impedance modulus and (b) reflection coefficient of the substrate (dashed black), of the loaded Bragg cell (solid red) and of the backing (dashed green).

\section{C.2. Electrical impedance}

Thickness variations [5] and attenuations in the periodic structure lead to damped parasitic resonances, while the input impedance of the backing is lowered in the frequency range of the piezoelectric resonance (Fig. 10). A nearly free resonance of the piezoelectric layer is obtained, equivalent to that obtained without any backing nor substrate.

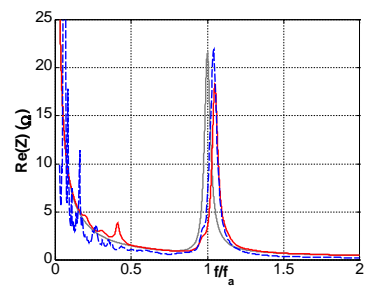

a)

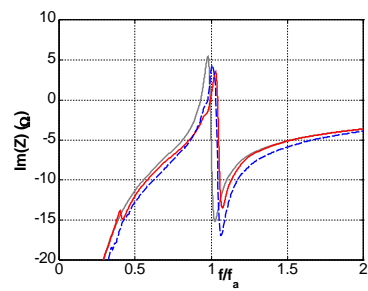

b)
Fig.10: (a) Real and (b) imaginary parts of the electrical impedance obtained by experiment (dashed blue) and modeling (solid red) of the integrated Bragg transducer based on the structure shown in Fig.8 in comparison with the fitted electrical impedance of the $\mathrm{Pz} 21$ piezoelectric layer with $\mathrm{Ag}$ electrodes (solid gray).

\section{C.3. Pulse-echo response}

Simulated pulse-echo response and related spectrum of such a Bragg transducer can then be improved by adding matching layers on the front face (Fig.11). The -6 $\mathrm{dB}$ bandwidth is then predicted around $30 \%$.

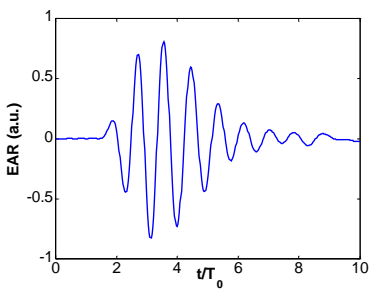

a)

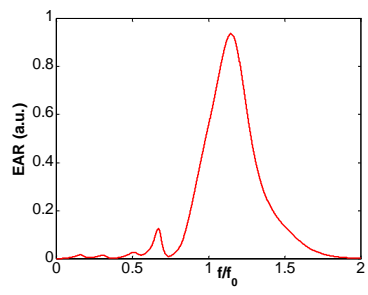

b)
Fig.11: KLM simulations of (a) pulse-echo response and (b) corresponding spectrum of the integrated Bragg transducer based on the structure shown in Fig.8, with two quarter wavelength matching layers on the front face.

\section{Conclusion}

The potential of a periodic structure as backing material for high frequency ultrasonic transducers was investigated. On the basis of a recursive calculation of reflection and transmission coefficients, the influence of the layer properties was evaluated. Then, two sets of realistic materials were proposed to constitute a basic stack. One sample was fabricated and its thickness profile was evaluated, showing strong variations around the mean value (up to $30 \%$ ). Nevertheless, the input impedance of the backing was lowered, resulting in a nearly free resonance of the piezoelectric layer. Finally, a transducer with improved sensitivity was simulated based on the screen-printed Bragg structure.

\section{E. Acknowledgments}

This work was supported by EC through MINUET project ( $6^{\text {th }} \mathrm{FP}$, Contract No. NMP2-CT-2004-505657).

\section{F. References}

[1] J.M. Conoir, "Acoustic scattering from cylinders" (in french), PhD thesis, Paris VI Univ., 1987.

[2] K.M. Lakin, K.T. McCarron and R.E. Rose, "Solidly mounted resonators and filters", IEEE Ultrasonics Symposium, p.905-908, 1995.

[3] S. Salgar, G. Kim, D.H. Han and B. Kim, "Modeling an simulation of the thin film bulk acoustic resonator", IEEE Ultrasonics Symposium, p.40-43, 2002.

[4] P. Maréchal, F. Levassort, J. Holc, L.P. TranHuu-Hue, M. Kosec and M. Lethiecq, "High frequency transducers based on integrated piezoelectric thick films for medical imaging", IEEE TUFFC, Vol. 53, p.15241533, 2006.

[5] C.J. Chung, Y.C. Chen, C.C. Cheng, C.L. Wei and K.S. Kao, "Influence of surface roughness of Bragg reflectors on resonance characteristics of solidly-mounted resonators", IEEE TUFFC, Vol. 54, p.802-808, 2007. 